\title{
AVERAGED CONTROLLABILITY OF THERMOELASTICITY EQUATIONS. AVERAGE STATE OF A RECTANGULAR PLATE
}

\author{
S. H. JILAVYAN ${ }^{1 *}$, As. Zh. KHURSHUDYAN ${ }^{2 * *}$ \\ ${ }^{1}$ Chair of Mechanics, YSU, Armenia \\ ${ }^{2}$ Institute of Mechanics, NAS of RA, Armenia
}

\begin{abstract}
The concept of averaged controllability has been introduced relatively recently aiming to analyse the controllability of systems or processes containing some important parameters that may affect the controllability in usual sense. The averaged controllability of various specific and abstract equations has been studied so far. Relatively little attention has been paid to averaged controllability of coupled systems. The averaged state of a thermoelastic rectangular plate is studied in this paper using the well-known Green's function approach. The aim of the paper is to provide a theoretical background for further exact and approximate controllability analysis of fully coupled thermoelasticity equations which will appear elsewhere.
\end{abstract}

MSC2010: Primary: 35Q93; Secondary: 74F05.

https://doi.org/10.46991/PYSU:A/2021.55.2.123

Keywords: averaged controllability, mathematical expectation, controllability of PDEs.

Introduction. hen dealing with controllability analysis of systems with uncertain or random parameters, it is important to take into account that the steering controls should not depend on these parameters. Looking at the usual (residual) definition of controllability, requiring to evaluate the residue [1]

$$
\mathcal{R}_{T}(u)=\left\|w\left(w_{0} ; u, T, \omega\right)-w_{T}\right\|,
$$

where $w$ is the system state, $w_{0}$ is the initial state, $u$ is the control, $T$ is the required control time, $\omega$ is a parameter, $w_{T}$ is a desired terminal state, it becomes obvious that any admissible control that may ensure $\mathcal{R}_{T}=0$ (exact controllability) or $\mathcal{R}_{T} \leq \varepsilon$ for a pre-defined tolerance $\varepsilon$ (approximate controllability), should depend on $\omega$. However, this is not feasible in the sense that the steering controls must depend only on pre-determined data.

\footnotetext{
* E-mail: samjilavyan@ysu.am

** E-mail: khurshudyan@mechins.sci.am
} 
In order to overcome this difficulty in the case when $\omega$ is uncertain, it has been suggested by the prominent mathematician Enrique Zuazua in [2] to use the averaged state

$$
\mathbb{A}[w]=\int w(\cdot ; \cdot, \cdot, \omega) \mathrm{d} \omega
$$

instead of $w$ itself. Integration goes over all possible values of $\omega$. Thus, the residue takes the form

$$
\mathcal{R}_{T}^{a v}(u)=\left\|\mathbb{A}[w]-w_{T}\right\| .
$$

Apparently, now $\mathcal{R}_{T}^{a v}$ does not depend on $\omega$, so does $u$ as well.

In the case when $\omega$ is a random variable, this difficulty is overcome, again by the suggestion of Enrique Zuazua [3], by considering the mathematical expectation of $w$,

$$
\mathbb{M}[w]=\int w(\cdot ; \cdot, \cdot, \omega) \rho(\omega) \mathrm{d} \omega,
$$

where $\rho$ is the probability density function of $\omega$. The case of multiple parameters is treated analogously.

The definition of exact and approximate controllability is given as above in terms of $\mathcal{R}_{T}^{a v}$. In this case, however, in order to distinguish the difference, the property is referred to as averaged controllability, in view of involvement of the averaged state. Specifically, when $\mathcal{R}_{T}^{a v}(u)=0$ for an admissible control $u$, then the system is called averaged exactly controllable, and when $\mathcal{R}_{T}^{a v}(u) \leq \varepsilon$, the system is called averaged approximate controllable. For other relevant studies see also [4-8].

The aim of the current research is to carry out averaged (exact and approximate) controllability analysis for thermoelasticity equations in the whole three-dimsnional space with five uniformly distributed random parameters: Lamé coefficients, density, thermal expansion coefficient, thermal diffusivity coefficient. The heat diffusion is controlled by a time-dependent intensity of heaters continuously distributed over a bounded domain within the thermoelastic space. Given any initial state, finite control time, the problem is in determination of steering controls providing a given desired value to the mathematical expectation of the deformed state of the body. Constructing the Green's function solution of the governing coupled system, the mathematical expectations of interest are evaluated in an explicit form making the averaged controllability analysis straightforward. In this first part of the paper, we present the average state of a thermoelastic rectangular plate, the controllability analysis of which will appear in the second part of the paper.

Problem Statement. Consider a linear thermoelastic material occupying a domain $\Omega \subseteq \mathbb{R}^{3}$. Assume that at the undeformed state, the temperature of $\Omega$ is everywhere 0. Assuming linear Duhamel-Neumann relations, the state of $\Omega$ is defined as the solution of the coupled system of partial differential equations [9]

$$
\begin{aligned}
& \mu \nabla^{2} \boldsymbol{u}+(\lambda+\mu) \operatorname{grad} \operatorname{div} \boldsymbol{u}=\rho \frac{\partial^{2} \boldsymbol{u}}{\partial t^{2}}+(3 \lambda+2 \mu) e_{t} \operatorname{grad} \Theta, \\
& \kappa \Delta \Theta=\frac{\partial \Theta}{\partial t}-\eta(t) \chi_{\Omega_{\eta}}(\boldsymbol{x}),
\end{aligned}
$$


where $\boldsymbol{u}$ is point displacement and $\Theta$ is the temperature of $\Omega, \lambda$ and $\mu$ are the Lamé coefficients, $e_{t}$ is the thermal extension coefficient, $\rho$ is the density, $\kappa$ is the thermal diffusivity of the material, $\eta$ is the intensity and $\Omega_{\eta} \subseteq \Omega$ is the spatial distribution of the heaters. The operators $\nabla, \Delta$, grad and div are defined as usual, $\chi_{\Omega_{\eta}}$ is the indicator function of $\Omega_{\eta}$. The intensity of the heaters, $\eta$, is considered to be controllable. Hereinafter, it is assumed that $\eta \in \mathcal{A}=\left\{\eta \in L^{2}[0, T],|\eta| \leq \eta_{0}, \operatorname{supp}(\eta) \subseteq[0, T]\right\}$.

In case when $\Omega$ is bounded, its sufficiently smooth boundary $\partial \Omega$ is assumed to be rigidly fixed and subjected to a known temperature, leading to the boundary conditions

$$
\boldsymbol{u}=0, \Theta=0, \boldsymbol{x} \in \partial \Omega, t \in \mathbb{R}_{+} .
$$

The initial state at $t=0$ is given by

$$
\boldsymbol{u}=\boldsymbol{u}_{0}, \frac{\partial \boldsymbol{u}}{\partial t}=\boldsymbol{u}_{1}, \Theta=\Theta_{0}, t=0, \boldsymbol{x} \in \Omega .
$$

Assuming that the material characteristics, i.e. $\lambda, \mu, e_{t}, \rho$, and $\kappa$ are uniformly distributed, independent random variables, the problem is to study the averaged (exact and approximate) controllability of (1) within a finite amount of time. Specifically, given any initial state $\left(\boldsymbol{u}_{0}, \Theta_{0}\right)$ and a control time $T>0$, describe the set of resolving controls providing

$$
\mathcal{R}_{T}^{a v}(\eta)=\left\|\mathbb{M}_{5}[\boldsymbol{u}](\boldsymbol{x}, T)-\boldsymbol{u}_{T}(\boldsymbol{x})\right\|_{L^{2}(\Omega)}^{2}+\left\|\mathbb{M}_{1}[\Theta](\boldsymbol{x}, T)-\Theta_{T}(\boldsymbol{x})\right\|_{L^{2}(\Omega)}^{2} .
$$

Here, $\left(\boldsymbol{u}_{T}, \Theta_{T}\right) \in L^{2}(\Omega)$ is the desired terminal state to be achieved. Corresponding to argument, $\mathbb{M}_{5}[\boldsymbol{u}]$ is a three-component vector, while $\mathbb{M}_{1}[\Theta]$ is a scalar. The subscript of the expectation symbol indicates on how many of the random variables the argument (i.e. $\boldsymbol{u}$ or $\Theta$ ) depends. Evidently, $\boldsymbol{u}$ depends on all 5 random parameters, while $\Theta$ depends only on $\kappa$.

Under the additional assumption that the Poisson's ratio $v$ accepts approximately same value for almost all isotropic materials, it is possible to reduce the number of parameters to 4 . Indeed, since

$$
\lambda=\frac{v}{(1+v)(1-2 v)} E, \quad \mu=\frac{1}{2(1+v)} E,
$$

where $E$ is the Young's modulus, $E$ can be considered as a random variable instead of the couple $(\lambda, \mu)$. Nonetheless, for the sake of generality, hereinafter, the case of random $\lambda$ and $\mu$ is considered.

The Green's Function Solution of (1). For the sake of simplicity, let $\mu=\omega_{1}$, $\lambda=\omega_{2}, \rho=\omega_{3}, e_{t}=\omega_{4}$, and $\kappa=\omega_{5}$, and denote $\boldsymbol{\omega}=\left\{\omega_{i}\right\}_{i=1}^{5}$. Let $\boldsymbol{\omega}$ be constrained in a rectangular box, i.e. $\boldsymbol{\omega}_{0} \leq \boldsymbol{\omega} \leq \boldsymbol{\omega}_{1}$, where $\boldsymbol{\omega}_{0}, \boldsymbol{\omega}_{1} \in \mathbb{R}_{+}^{5}$ are given constant vectors. In order to determine the expectations $\mathbb{M}_{5}[\boldsymbol{u}]$ and $\mathbb{M}_{1}[\Theta]$ entering into (4) in terms of the random parameters, it is convenient to use the Green's function solution of (1)-(3). Since the second equation in (1) is merely for $\Theta$, its solution is represented as follows [10]:

$$
\Theta\left(\boldsymbol{x}, t ; \omega_{5}\right)=\int_{\Omega} \int_{0}^{t} G_{\Theta}\left(\boldsymbol{x}, \boldsymbol{\xi}, t-\tau ; \omega_{5}\right)\left[\Theta_{0}(\boldsymbol{\xi}) \boldsymbol{\delta}(\tau)+\eta(\tau) \chi_{\Omega_{\eta}}(\boldsymbol{\xi})\right] \mathrm{d} \boldsymbol{\xi} \mathrm{d} \tau .
$$


Here $\delta$ is the Dirac function,

$$
G_{\Theta}\left(\boldsymbol{x}, \boldsymbol{\xi}, t ; \omega_{5}\right)=G_{\Theta 1}\left(x_{1}, \xi_{1}, t ; \omega_{5}\right) G_{\Theta 2}\left(x_{2}, \xi_{2}, t ; \omega_{5}\right) G_{\Theta 3}\left(x_{3}, \xi_{3}, t ; \omega_{5}\right),
$$

where $\boldsymbol{x}=\left(\begin{array}{lll}x_{1} & x_{2} & x_{3}\end{array}\right), \boldsymbol{\xi}=\left(\begin{array}{lll}\xi_{1} & \xi_{2} & \xi_{3}\end{array}\right)$ and $G_{\Theta i}$ are the Green's functions of the corresponding one-dimensional boundary-value problems.

In order to determine the Green's function representation for $\boldsymbol{u}$, notice that in view of absence of body forces and that the linear Duhamel-Neumann relations are accepted excluding rotations, there exist a scalar function $\Phi$ such that

$$
\boldsymbol{u}=\operatorname{grad} \Phi \quad \text { in } \Omega \text {. }
$$

Moreover, $\Phi$ describes the waves of extension and satisfies the wave equation

$$
\frac{2 \omega_{1}+\omega_{2}}{\omega_{3}} \Delta \Phi=\frac{\partial^{2} \Phi}{\partial t^{2}}+\frac{2 \omega_{1}+3 \omega_{2}}{\omega_{3}} \omega_{4} \Theta,
$$

subject to the boundary condition (cf. (2))

$$
\operatorname{grad} \Phi=0, \quad x \in \partial \Omega, t \in \mathbb{R}_{+},
$$

and the initial conditions (cf. (3))

$$
\Phi=\Phi_{0}, \frac{\partial \Phi}{\partial t}=\Phi_{1}, t=0, x \in \Omega,
$$

where $\boldsymbol{u}_{p}=\operatorname{grad} \Phi_{p}, p=0,1$.

It is well-known that the solution of (7)-(9) is determined in terms of the Green's function as follows [10]:

$$
\begin{aligned}
\Phi(\boldsymbol{x}, t ; \boldsymbol{\omega}) & =\int_{\Omega} \int_{0}^{t} G_{\Phi}(\boldsymbol{x}, \boldsymbol{\xi}, t-\tau ; \hat{\boldsymbol{\omega}})\left[\Phi_{0}(\boldsymbol{\xi}) \dot{\boldsymbol{\delta}}(\tau)+\Phi_{1}(\boldsymbol{\xi}) \boldsymbol{\delta}(\tau)\right. \\
& \left.+\left(2 \omega_{1}+3 \omega_{2}\right) \frac{\omega_{4}}{\omega_{3}} \Theta\left(\boldsymbol{\xi}, \tau ; \omega_{5}\right)\right] \mathrm{d} \boldsymbol{\xi} \mathrm{d} \tau,
\end{aligned}
$$

where $\hat{\omega}=\left\{\omega_{i}\right\}_{i=1}^{3}$. Once $\Phi$ is determined, the displacement field $\boldsymbol{u}$ is evaluated accoring to (6).

The Case of Rectangular Parallelepiped. Green's function for one-, two- and three-dimensional heat and wave equations for bounded and unbounded $\Omega$ and for various initial/boundary conditions can be found in the handbook [10]. For example, when $\Omega=\left\{\boldsymbol{x} \in \mathbb{R}^{3}, 0 \leq x_{i} \leq l_{i}, i=1,2,3\right\}$ is a rectangular parallelepiped, then [10]

$$
G_{\Theta}\left(\boldsymbol{x}, \boldsymbol{\xi}, t ; \omega_{5}\right)=\prod_{i=1}^{3} \frac{2}{l_{i}} \sum_{n=1}^{\infty} \varphi_{n i}\left(x_{i}\right) \varphi_{n i}\left(\xi_{i}\right) \exp \left[-\omega_{5} \lambda_{n i}^{2} t\right], \varphi_{n i}\left(x_{i}\right)=\sin \left(\lambda_{n i} x_{i}\right),
$$

and

$$
\begin{aligned}
G_{\Phi}(\boldsymbol{x}, \boldsymbol{\xi}, t ; \hat{\boldsymbol{\omega}}) & =\frac{1}{l_{1} l_{2} l_{3}}\left[t+\sqrt{\frac{\omega_{3}}{2 \omega_{1}+\omega_{2}}}\right. \\
& \left.\times \sum_{n_{1}, n_{2}, n_{3}=0}^{\infty} \frac{\alpha_{n_{1}} \alpha_{n_{2}} \alpha_{n_{3}}}{\sigma_{n_{1} n_{2} n_{3}}} \sin \left(\sigma_{n_{1} n_{2} n_{3}} \sqrt{\frac{2 \omega_{1}+\omega_{2}}{\omega_{3}}} t\right) \prod_{i=1}^{3} \psi_{n_{i}}\left(x_{i}\right) \psi_{n_{i}}\left(\xi_{i}\right)\right],
\end{aligned}
$$




$$
\begin{gathered}
\alpha_{n_{i}}=\left\{\begin{array}{ll}
1, & n_{i}=0, \\
2, & n_{i} \geq 1,
\end{array} \quad \sigma_{n_{1} n_{2} n_{3}}=\sqrt{v_{n_{1}}^{2}+v_{n 2}^{2}+v_{n 3}^{2}},\right. \\
\lambda_{n i}=\frac{\pi n}{l_{i}}, v_{n_{i}}=\frac{\pi n_{i}}{l_{i}}, \psi_{n_{i}}\left(x_{i}\right)=\cos \left(\lambda_{n i} x_{i}\right) .
\end{gathered}
$$

Determination of the Averaged State. In view of the obvious relation

$$
\mathbb{M}_{5}[\boldsymbol{u}]=\operatorname{grad} \mathbb{M}_{5}[\Phi]
$$

the expectation $\mathbb{M}_{5}[\Phi]$ will be evaluated below. Taking into account that both $\mathbb{M}_{5}$ and $\mathbb{M}_{1}$ are invariant with respect to the integral integral sign, it is obtained

$$
\begin{aligned}
\mathbb{M}_{5}[\Phi](\boldsymbol{x}, t) & =\int_{\Omega} \int_{0}^{t}\left[\mathbb{M}_{3}\left[G_{\Phi}\right](\boldsymbol{x}, \boldsymbol{\xi}, t-\tau)\left[\Phi_{0}(\boldsymbol{\xi}) \dot{\boldsymbol{\delta}}(\tau)+\Phi_{1}(\boldsymbol{\xi}) \boldsymbol{\delta}(\tau)\right]\right. \\
& \left.+\mathbb{M}_{5}\left[\left(2 \omega_{1}+3 \omega_{2}\right) \frac{\omega_{4}}{\omega_{3}} G_{\Phi} \Theta\right](\boldsymbol{x}, \boldsymbol{\xi}, t, \tau)\right] \mathrm{d} \boldsymbol{\xi} \mathrm{d} \tau \\
\mathbb{M}_{1}[\Theta](\boldsymbol{x}, t)= & \int_{\Omega} \int_{0}^{t} \mathbb{M}_{1}\left[G_{\Theta}\right](\boldsymbol{x}, \boldsymbol{\xi}, t-\tau)\left[\Theta_{0}(\boldsymbol{\xi}) \delta(\tau)+\eta(\tau) \chi_{\Omega_{\eta}}(\boldsymbol{\xi})\right] \mathrm{d} \boldsymbol{\xi} \mathrm{d} \tau
\end{aligned}
$$

Since $\omega_{i}, i=1, \ldots, 5$, are uniformly distributed random variables and $G_{\Theta}$ depends only on $\omega_{5}$, then

$$
\mathbb{M}_{1}\left[G_{\Theta}\right](\boldsymbol{x}, \boldsymbol{\xi}, t)=\frac{1}{\omega_{51}-\omega_{50}} \int_{\omega_{50}}^{\omega_{51}} G_{\Theta}\left(\boldsymbol{x}, \boldsymbol{\xi}, t ; \omega_{5}\right) \mathrm{d} \omega_{5},
$$

On the other hand,

$$
\begin{gathered}
\mathbb{M}_{3}\left[G_{\Phi}\right](\boldsymbol{x}, \boldsymbol{\xi}, t)=\frac{1}{A_{3}} \int_{\hat{\boldsymbol{\omega}}_{0}}^{\hat{\omega}_{1}} G_{\Phi}(\boldsymbol{x}, \boldsymbol{\xi}, t ; \hat{\boldsymbol{\omega}}) \mathrm{d} \hat{\boldsymbol{\omega}} \\
\mathbb{M}_{5}\left[G_{\Phi}\right](\boldsymbol{x}, \boldsymbol{\xi}, t)=\frac{1}{A_{5}} \int_{\omega_{0}}^{\omega_{1}}\left(2 \omega_{1}+3 \omega_{2}\right) \frac{\omega_{4}}{\omega_{3}} G_{\Phi}(\boldsymbol{x}, \boldsymbol{\xi}, t ; \hat{\boldsymbol{\omega}}) \Theta\left(\boldsymbol{\xi}, \tau ; \omega_{5}\right) \mathrm{d} \boldsymbol{\omega},
\end{gathered}
$$

where $\hat{\boldsymbol{\omega}}_{p}=\left\{\omega_{p i}\right\}_{i=1}^{3}, p=0,1$,

$$
\begin{gathered}
A_{3}=\left(\omega_{11}-\omega_{10}\right)\left(\omega_{21}-\omega_{20}\right)\left(\omega_{31}-\omega_{30}\right), \\
A_{5}=A_{3}\left(\omega_{41}-\omega_{40}\right)\left(\omega_{51}-\omega_{50}\right) .
\end{gathered}
$$

A Particular Case. In particular, in the case considered in Section, straightforward integration in (13) and (14) provides

$$
\begin{gathered}
\mathbb{M}_{1}\left[G_{\Theta}\right](\boldsymbol{x}, \boldsymbol{\xi}, t)=\prod_{i=1}^{3} \frac{2}{l_{i}} \sum_{n=1}^{\infty} \frac{\exp \left[-\omega_{51} \lambda_{n i}^{2} t\right]-\exp \left[-\omega_{50} \lambda_{n i}^{2} t\right]}{\lambda_{n i}^{2} t\left(\omega_{51}-\omega_{50}\right)} \varphi_{n i}\left(x_{i}\right) \varphi_{n i}\left(\xi_{i}\right) . \\
\mathbb{M}_{3}\left[G_{\Phi}\right](\boldsymbol{x}, \boldsymbol{\xi}, t)=\frac{t}{l_{1} l_{2} l_{3}}+\frac{1}{A l_{1} l_{2} l_{3}} \sum_{n_{1}, n_{2}, n_{3}=0}^{\infty} \frac{\alpha_{n_{1}} \alpha_{n_{2}} \alpha_{n_{3}}}{\sigma_{n_{1} n_{2} n_{3}}} I_{n_{1} n_{2} n_{3}}(t) \prod_{i=1}^{3} \psi_{n_{i}}\left(x_{i}\right) \psi_{n_{i}}\left(\xi_{i}\right),
\end{gathered}
$$


where

$$
I_{n_{1} n_{2} n_{3}}(t)=\int_{\hat{\omega}_{0}}^{\hat{\omega}_{1}} \sqrt{\frac{\omega_{3}}{2 \omega_{1}+\omega_{2}}} \sin \left(\sigma_{n_{1} n_{2} n_{3}} \sqrt{\frac{2 \omega_{1}+\omega_{2}}{\omega_{3}}} t\right) \mathrm{d} \hat{\boldsymbol{\omega}} .
$$

Note that $\mathbb{M}_{5}\left[\left(2 \omega_{1}+3 \omega_{2}\right) \frac{\omega_{4}}{\omega_{3}} G_{\Phi} \Theta\right]$ is evaluated similarly. Its final form is of burdensome length and is not brought here. Note only that as a result of integration with respect to $\omega$, the following quantity arises:

$$
\begin{aligned}
J_{n n_{1} n_{2} n_{3}}(t)=\int_{\omega_{0}}^{\omega_{1}}\left(2 \omega_{1}+3 \omega_{2}\right) & \frac{\omega_{4}}{\omega_{3}} \sqrt{\frac{\omega_{3}}{2 \omega_{1}+\omega_{2}}} \sin \left(\sigma_{n_{1} n_{2} n_{3}} \sqrt{\frac{2 \omega_{1}+\omega_{2}}{\omega_{3}}} t\right) \\
& \times \exp \left[-\omega_{5} \lambda_{n i}^{2} t\right] \mathrm{d} \omega .
\end{aligned}
$$

The integrals $I_{n_{1} n_{2} n_{3}}$ and $J_{n n_{1} n_{2} n_{3}}$ can be obtained explicitly. The definite integral in $I_{n_{1} n_{2} n_{3}}$ is evaluated as follows:

$$
\begin{aligned}
\int \sqrt{\frac{\omega_{3}}{2 \omega_{1}+\omega_{2}}} \sin \left(\sqrt{\frac{2 \omega_{1}+\omega_{2}}{\omega_{3}}} \sigma t\right) \mathrm{d} \hat{\omega} \\
\quad=\frac{1}{36 \sigma^{3} t^{3}}\left[-\left(2 \omega_{1}+\omega_{2}\right)^{3} \sigma^{6} t^{6} \mathrm{Ci}\left(\sqrt{\frac{2 \omega_{1}+\omega_{2}}{\omega_{3}}} \sigma t\right)\right. \\
+\sigma t \sqrt{\left(2 \omega_{1}+\omega_{2}\right) \omega_{3}}\left[\left(\left(2 \omega_{1}+\omega_{2}\right) \sigma^{2} t^{2}-\omega_{3}\right)^{2}+23 \omega_{3}^{2}\right] \sin \left(\sqrt{\frac{2 \omega_{1}+\omega_{2}}{\omega_{3}}} \sigma t\right) \\
\left.+\omega_{3}\left[\left(\left(2 \omega_{1}+\omega_{2}\right) \sigma^{2} t^{2}-3 \omega_{3}\right)^{2}-33 \omega_{3}^{2}\right] \cos \left(\sqrt{\frac{2 \omega_{1}+\omega_{2}}{\omega_{3}}} \sigma t\right)\right]
\end{aligned}
$$

where $\mathrm{Ci}$ is the integral cosine.

When evaluating the definite integral in $J_{n n_{1} n_{2} n_{3}}$, it is easy to see that the integrand is linear in $\omega_{4}$ and exponential in $\omega_{5}$. The difficult part is in integrating with respect to $\hat{\omega}$. Eventually, the integral is evaluated as follows:

$$
\begin{aligned}
\int\left(2 \omega_{1}+3 \omega_{2}\right) \frac{\omega_{4}}{\omega_{3}} \sqrt{\frac{\omega_{3}}{2 \omega_{1}+\omega_{2}}} \sin \left(\sigma \sqrt{\frac{2 \omega_{1}+\omega_{2}}{\omega_{3}}} t\right) \exp \left[-\omega_{5} \lambda^{2} t\right] \mathrm{d} \omega \\
=-\frac{\omega_{4}^{2} \exp \left[-\omega_{5} \lambda^{2} t\right]}{12 \lambda \sigma^{5} t^{6}}\left[f_{1}(t ; \hat{\omega}) \cos \left(\sqrt{\frac{2 \omega_{1}+\omega_{2}}{\omega_{3}}} \sigma t\right)\right. \\
\left.+f_{2}\left(t, \omega_{1}, \omega_{2}\right) \operatorname{Ci}\left(\sqrt{\frac{2 \omega_{1}+\omega_{2}}{\omega_{3}}} \sigma t\right)-f_{3}(t ; \hat{\omega}) \sin \left(\sqrt{\frac{2 \omega_{1}+\omega_{2}}{\omega_{3}}} \sigma t\right)\right],
\end{aligned}
$$

where

$$
\begin{aligned}
f_{1}(t ; \hat{\omega})= & \omega_{3}\left(\sigma^{4} t^{4}\left(2 \omega_{1}+\omega_{2}\right)\left(2 \omega_{1}-5 \omega_{2}\right)-6 \sigma^{2} t^{2}\left(10 \omega_{1}+7 \omega_{2}\right) \omega_{3}+96 \omega_{3}^{2}\right) \\
& f_{2}\left(t ; \omega_{1}, \omega_{2}\right)=\sigma^{6} t^{6}\left(2 \omega_{1}+\omega_{2}\right)^{2}\left(2 \omega_{1}-5 \omega_{2}\right) \\
f_{3}(t ; \hat{\omega})= & \sigma t \sqrt{\left(2 \omega_{1}+\omega_{2}\right) \omega_{3}} \\
\times & \left(\sigma^{4} t^{4}\left(2 \omega_{1}+\omega_{2}\right)\left(2 \omega_{1}-5 \omega_{2}\right)-2 \sigma^{2} t^{2}\left(2 \omega_{1}-5 \omega_{2}\right) \omega_{3}-96 \omega_{3}^{2}\right)
\end{aligned}
$$


Conclusion. Various types of controllability has been introduced to study possibilities of providing required states for systems and processes in some broad or narrow sense. At that, a special attention is paid to the sensitivity of the controllability with respect to characteristic parameters of the system or process under study.

In this paper, we derive the averaged state of a rectangular, linear thermoelastic plate heated by an external heat source with given location and controllable intensity. The averaging parameters are the Lamé coefficients, density, thermal expansion coefficient and thermal diffusivity of the plate. Using the well-known Green's function approach developed in [1], the dependence of the average state on parameter values is made explicit, simplifying the average controllability analysis much further.

Future work will include the study of exact and approximate controllability of the plate and will appear elsewhere.

Received 12.02.2021

Reviewed 08.07.2021

Accepted 21.07.2021

\section{R E F E R E N C E S}

1. Avetisyan A.S., Khurshudyan As.Zh. Controllability of Dynamic Systems: The Green's Function Approach. Cambridge Scholars Publishing, Cambridge (2018).

2. Zuazua E. Averaged Control. Automatica 50 (2014), 3077-3087. https://doi.org/10.1016/j.automatica.2014.10.054

3. Lü Q., Zuazua E. Averaged Controllability for Random Evolution Partial Differential Equations. Journal de Mathématiques Pures et Appliquées 105 (2016), 367-414. https://doi.org/10.1016/j.matpur.2015.11.004

4. Lazar M., Zuazua E. Averaged Control and Observation of Parameter Depending Wave Equations. Comptes Rendus Mathematique 352 (2014), 497-502. https://doi.org/10.1016/j.crma.2014.04.007

5. Lohéac J., Zuazua E. Averaged Controllability of Parameter Dependent Conservative Semigroups. J. Diff. Equat. 262 (2017), 1540-1574. https://doi.org/10.1016/j.jde.2016.10.017

6. Lü Q., Zuazua E. Averaged Controllability for Parameter Dependent Evolution Partial Differential Equations. Open Problems in Systems and Control. IMA-Minneapolis (2017), $1-4$.

7. Klamka J., Khurshudyan As.Zh. Averaged Controllability of Heat Equation in Unbounded Domains with Uncertain Geometry and Location of Controls: The Green's Function Approach. Archives of Control Sciences 29 (LXV) (2019), 573-584. https://doi.org/10.24425/acs.2019.131226

8. Avetisyan A.S., Khurshudyan As.Zh. Averaged Controllability of Euler Bernoulli Beam Structures with Random Parameters: The Green's Function Approach. Proceedings of NAS of Armenia. Mechanics 72 : 4 (2019), 7-18. https://doi.org/10.33018/72.4.1

9. Nowacki W. Thermoelasticity (2nd ed.). Pergamon, Oxford (1986). 
10. Polyanin A.D., Nazaikinski V.E. Handbook of Linear Partial Differential Equations (2nd ed.). Chapman \& Hall/CRC Press, Boca Raton (2016).

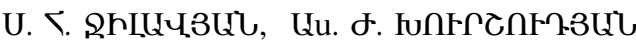

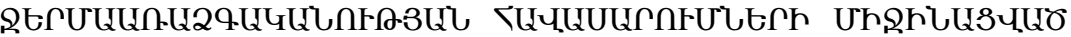

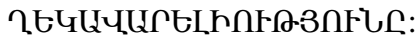

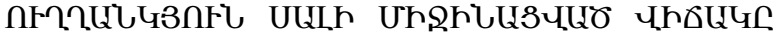

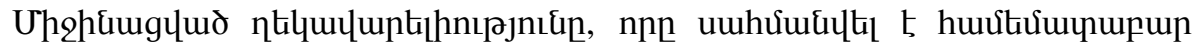

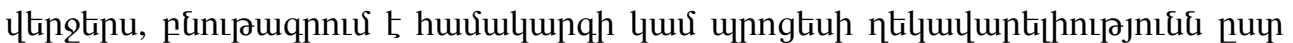

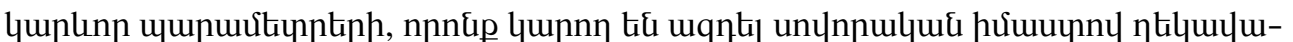

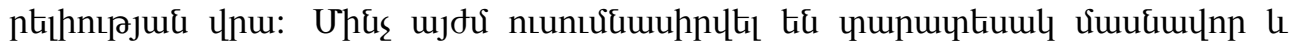

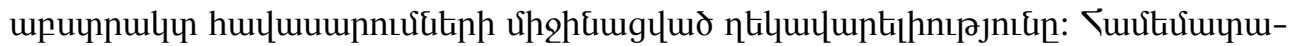

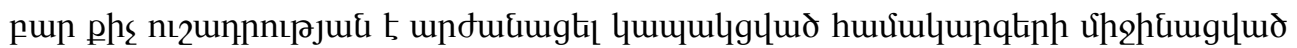

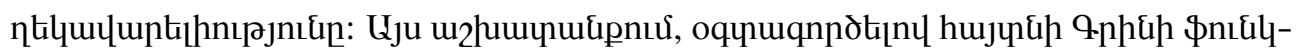

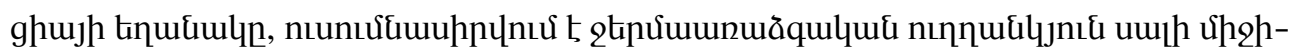

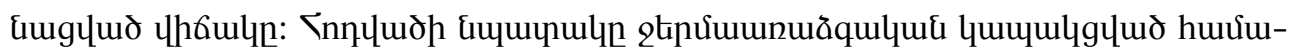

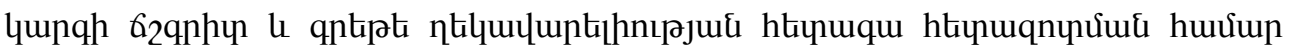

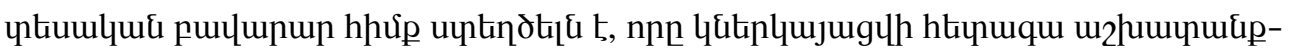
Gitinnư:

\title{
с. А. ДЖИЛАВЯН, Ас. Ж. ХУРШУДЯН
}

\section{УСРЕДНЕННАЯ УПРАВЛЯЕМОСТЬ УРАВНЕНИЙ ТЕРМОУПРУГОСТИ. УСРЕДНЕННОЕ СОСТОЯНИЕ ПРЯМОУГОЛЬНОЙ ПЛАСТИНКИ}

Понятие усредненной управляемости было введено относительно недавно с целью анализа управляемости систем или процессов, содержащих некоторые важные параметры, которые могут влиять на управляемость в обычном смысле. До сих пор изучалась усредненная управляемость различных конкретных и абстрактных уравнений. Относительно мало внимания уделяется усредненной управляемости связанных систем. В данной работе изучается усредненное состояние термоупругой прямоугольной пластинки с использованием известного подхода - функции Грина. Цель статьи - построить теоретическую основу для дальнейшего точного и приближенного анализа управляемости полностью связанных уравнений термоупругости, результаты которого будут опубликованы в дальнейшем. 